\title{
CAMBIAMENTI CLIMATICI: EVIDENZE SCIENTIFICHE, RISCHI ED OPPORTUNITÀ DI MITIGAZIONE
}

\author{
FILIPPO GIORGI (*)
}

Nota presentata dal m.e. C.E. Bottani

(Adunanza del 24 maggio 2018)

SuNTO. - Questo contributo presenta le molteplici evidenze che portano la comunità scientifica a concludere che il riscaldamento globale è in atto ed è dovuto per la maggior parte alle emissioni di gas serra, principalmente anidride carbonica e metano, derivanti dall'uso di combustibili fossili ed alcune pratiche agricole intensive. I principali cambiamenti climatici associati al riscaldamento globale sono poi discussi, insieme con gli scenari di clima futuro sviluppati con modelli climatici globali e gli impatti che i cambiamenti climatici possono avere su alcuni settori socioeconomici. Infine il contributo descrive le politiche di risposta al riscaldamento globale, ed in particolare i concetti di adattamento e mitigazione (riduzione delle emissioni di gas serra).

$$
* * *
$$

ABSTRACT. - This contribution presents the various pieces of evidence which bring the scientific community to conclude that global warming is happening and it is mostly due to anthropogenic emissions of greenhouse gases, mainly carbon dioxide and methane, deriving from the use of fossil fuels and some intensive agricultural practices. The main climatic changes associated with global warming are then discussed, along with the main model-derived future climate scenarios and the impacts that climate change can have on different socioeconomic sectors. Finally, the response policies to global warming are described, and in particular the concepts of adaptation and mitigaziotn (reduction of greenhouse gas emissions).

(*) Abdus Salam International Centre for Theoretical Physics, Trieste, Italy. E-mail: giorgi@ictp.it 


\section{INTRODUZIONE}

Dall'inizio dell'era industriale le attività umane hanno cominciato a modificare il pianeta Terra al punto tale che molti ritengono che siamo entrati in una nuova era che possiamo definire dell'Antropocene [1]. Attività come agricultura, urbanizzazione e deforestazione hanno modificato profondamente la superficie dei continenti, tanto che ormai sono molto limitate le aree del globo che possiamo veramente considerare allo stato "naturale". Ogni anno attività industriali, agricole, e domestiche immettono in atmosfera enormi quantità di agenti chimici, che portano alla produzione di sostanze dannose alla salute umana e agli ecosistemi, come l'ozono ed il particolato atmosferico. Immagini da satellite, per esempio, ci mostrano come aree quali l'Asia meridionale ed orientale siano avvolte quasi costantemente in una cappa di inquinamento. Un inquinamento che non risparmia neanche le aree più remote del globo, come l'Artico e l'Antartide, e tanto meno gli oceani, divenuti ormai il ricettacolo di enormi quantità di rifiuti prodotti dalle nostre aree agricole ed urbane.

Ma la manifestazione più impressionante dell'Antropocene è sicuramente il riscaldamento globale ed i cambiamenti climatici ad esso associati. Le crescenti emissioni di gas ad "effetto serra", o "gas serra" dovute ad attività umane hanno portato ad un aumento delle temperature globali superficiali di oltre un grado centigrado dall'inizio del 20mo secolo [2], un riscaldamento che, come vedremo, nel contesto degli ultimi 11,000 anni è di una rapidità assolutamente inaudita [3]. Questo sta portando a tutta una serie di modifiche del clima che, se dovessero continuare al ritmo attuale per le prossime decadi, avrebbero effetti sulla società che ne potrebbero mettere in pericolo lo sviluppo sostenibile. Nonostante le evidenze del riscaldamento globale e delle sue cause siano ormai schiaccianti, i governi sono ancora molto riluttanti ad implementare politiche efficaci tese a contrastare i cambiamenti climatici. Le cause di questa mancanza di azione sono di varia natura, ma è chiaro che un primo passo importante in questo contesto è la conoscenza di quello che oggi la scienza sa, e non sa, sul tema dei cambiamenti climatici.

Lo scopo di questo contributo è quindi quello di presentare e discutere in maniera sintetica, ma scientificamente rigorosa, le questioni fondamentali riguardanti il problema del riscaldamento globa- 
le, dei cambiamenti climatici ad esso associati, degli effetti sulla società e delle politiche necessarie a contrastare questo fenomeno. Questo è fatto nella forma di risposte a quelle che sono le domande fondamentali su questo tema che emergono nel corso del dibattito sul riscaldamento globale. Le domande sono affrontate in maniera sequenziale nelle prossime sezioni di questo contributo perchè l'una scaturisce naturalmente dalla risposta a quella precedente.

Da notare che le tematiche di questo contributo sono trattate in maniera più estesa e comprensiva nel libro "L'Uomo e la Farfalla. Sei domande su cui Riflettere per Comprendere i Cambiamenti Climatici" edito dalla FrancoAngeli Editore, uscito nel 2018 [4]. Infine, i dati su cui è basata la discussione sono per lo più ottenuti dai rapporti redatti dall'Intergovernmental Panel on Climate Change nel corso delle ultime 3 decadi.

\section{IL RISCALDAMENTO GLOBALE È IN ATTO?}

La domanda fondamentale sui cambiamenti climatici è se il riscaldamento globale sia effettivamente in atto. Questo tema è stato dibattuto per molti anni, ma ormai una molteplicità di evidenze prova in maniera categorica e condivisa all'interno della comunità scientifica che la risposta a questa domanda è affermativa. La prima evidenza è l'andamento della stima delle temperature globali in superficie, stima che viene ottenuta mettendo insieme ed omogeneizzando dati da decine di migliaia di stazioni meteo, navi, boe etc. Diversi laboratori nel mondo producono regolarmente queste stime di temperatura globale, ed una di queste (prodotta dalla NASA) è mostrata in Fig. 1. Anche se esistono delle piccole differenze fra le stime prodotte da diversi gruppi di ricerca, tutte concordano nel rilevare che dall'inizio del 20 mo secolo la temperatura media globale superficiale è aumentata di poco più di $1^{\circ} \mathrm{C}$, e che l'ultima decade è stata di gran lunga la più calda negli ultimi 120 anni. È importante notare in Fig. 1 che esistono delle oscillazioni nella temparatura globale, sia da un anno all'altro che da una decade all'altra, sovrapposte al trend di riscaldamento. Queste sono dovute alla variabilità naturale del sistema climatico, che è un sistema complesso e non-lineare caratterizzato da un comportamento di tipo caotico. 


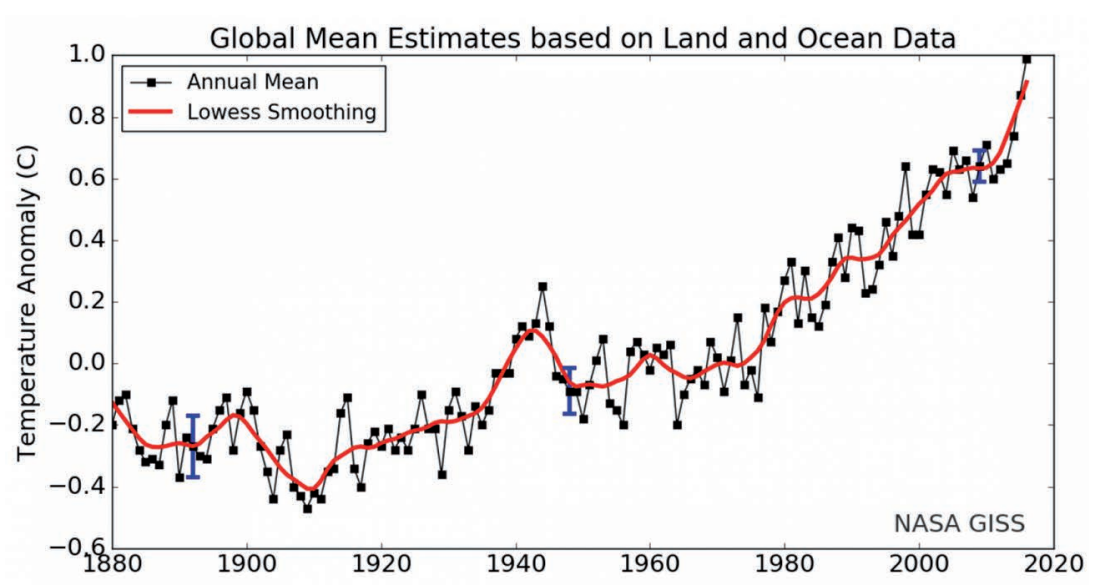

Fig. 1. Anomalie annuali di temperatura superficiale globale (rispetto al periodo di riferimento 1940-1970) dal 1880 al 2016. La curva indica una media corrente di 5 anni. Le tre piccole barre verticali indicano l'incertezza sulla stima delle medie in diversi periodi del record storico. (Cortesia della NASA-GISS).

Una seconda evidenza del riscaldamento globale è la fusione dei ghiacciai continentali, che sta avvenendo nella maggior parte dei ghiacciai del pianeta [5]. Per esempio, il monitoraggio dei ghiacciai Alpini mostra che tutti questi sono in fase di recessione. Particolamente preoccupante è poi la fusione dei ghiacci della Groenlandia, che sta avvenendo a ritmi molto maggiori di quelli stimati dai modelli glaciologici, probabilmente a causa di un effetto di scurimento dei ghiacci da parte del particolato atmosferico proveniente da Europa, America ed Asia [6]. Non solo i ghiacci continentali stanno fondendo, ma anche i ghiacci marini dell'Artico. Immagini da satellite mostrano in maniera impressionante come a fine estate (il periodo di minore copertura di ghiacci artici) negli anni 80 quasi l'intera calotta Artica era coperta di ghiacci, mentre già dagli ultimi anni circa la metà della calotta Artica rimane scoperta, e molti prevedono che fra qualche decade avremo un Artico senza ghiacci a fine estate. In Antartide le temperature sono troppo basse per causare la fusione dei ghiacci sul continente, però un altro preoccupante fenomeno è in atto. Le zone costiere dell'Antartide Occidentale sono caratterizzate dalla presenza di enormi lingue di ghiaccio che si estendono dalle coste sull'oceano (chiamate in inglese "ice -shelves"). Nelle ultime decadi diverse di queste ice-shelves, di estensione pari a migliaia di $\mathrm{km}^{2}$ si sono sgretolate con eventi della 
durata di poche settimane, per esempio nel Febbraio del 2002 [7], riversando nel mare un enorme numero di icebergs. Il motivo principale è il riscaldamento delle acque oceaniche sottostanti le ice-shelves, che ne indebolisce la struttura. Infatti, dati da navi e boe, in particolare la densa rete di boe ARGO attiva dagli anni 2000, mostrano come anche gli oceani si stiano riscaldando, almeno fino ad una profondità di 2000 $\mathrm{m}$ (un'altra evidenza del riscaldamento globale) [8].

L'acqua proveniente dalla fusione dei ghiacci continentali e l'espansione termica degli oceani sono alla base di una ulteriore evidenza del riscaldamento globale, e cioè l'innalzamento del livello del mare. Osservazioni basate sia da rilevamenti costieri che, più recentemente, da dati da satellite (come il TOPEX-POSEIDON), mostrano che il livello del mare globale è aumentato di circa $25 \mathrm{~cm}$ negli ultimi 120 anni [8]. Il contributo maggiore a questo innalzamento è dato dalla fusione dei ghiacci continentali, ma il contributo che sta crescendo alla maggiore velocità è quello della fusione dei ghiacci della Groenlandia.

Infine, un'ultima evidenza è l'aumento delle temperature in troposfera, i primi $\sim 15 \mathrm{~km}$ dell'atmosfera terrestre. Le temperature troposferiche possono essere misurate o da palloni sonda o da dati da satellite inseriti in modelli di trasporto radiativo. Mentre le prime stime da dati satellitari fatte negli anni 2000 non sembravano mostrare un riscaldamento, una migliore calibrazione di questi dati in studi più recenti mostra un riscaldamento pienamente in linea con quello superficiale [9].

Tutte le evidenze descritte in questa sezione hanno quindi portato la comunità scientifica a dichiarare che il riscaldamento globale in atto è inequivocabile [2].

\section{COSA STA CAUSANDO IL RISCALDAMENTO GLOBALE?}

Avendo stabilito che siamo in un trend di riscaldamento globale, la domanda che ne segue è cosa lo stia causando. Il clima della Terra può variare per tre tipi di cause: l'effetto di forzanti esterni naturali; l'effetto di forzanti esterni derivanti da attività umane (o forzanti antropici); e la variabilità naturale del sistema climatico.

Un tipico esempio della prima categoria è quello dei cosidetti cicli di Milankovic. Nel corso dell'ultimo milione di anni c'è stato un alternarsi abbastanza regolare di periodi glaciali ed interglaciali con periodi varianti dai $\sim 120,000$ ai 20,000 anni. L'ultimo picco glaciale è occorso 
circa 21,000 anni fa, quando le temperature hanno cominciato lentamente a risalire fino a raggiungere il picco interglaciale caldo circa 10,000 anni fa. Le temperature globali sono state poi stabili per circa 5000 anni, quando è cominciata la discesa molto lenta verso il prossimo periodo glaciale, che normalmente dovrebbe essere raggiunto fra qualche decina di migliaia di anni. Il forzante esterno naturale che determina questi cicli è la variazione regolare di alcune caratteristiche dell'orbita terrestre, come l'eccentricità dell'orbita, l'inclinazione dell'asse terrestre e la precessione dell'orbita. Queste variazioni però hanno luogo su scale temporali di decine di migliaia di anni e quindi non sono rilevanti per il nostro problema dei cambiamenti climatici su scale che vanno da alcune decine a centinaia di anni. A queste scale i forzanti esterni più importanti sono le variazioni interdecadali della intensità della radiazione solare e l'effetto di grandi eruzioni vulcaniche che immettono nell'alta atmosfera enormi quantità di particolato atmosferico, dove il particolato può risiedere anche per anni e influenzare il budget energetico dell'atmosfera con la riflessione e assorbimento della radiazione solare.

Il forzante antropico più importante è legato all'effetto dei cosidetti gas serra. Questi hanno la proprietà di assorbire parte della radiazione infrarossa emessa dalla superficie terrestre e riemetterla in tutte le direzioni, quindi verso lo spazio ma anche verso la superficies stessa, intrappolando quindi parte di questa energia e riscaldando la superficie e la bassa atmosfera. I più importanti gas serra di origine antropica sono l'anidride carbonica (CO2), che è principalmente un prodotto della combustione, ed il metano $(\mathrm{CH} 4)$, che è un prodotto della digestione. Prima dell'inizio dell'era industriale (metà del 19mo secolo) le concentrazioni atmosferiche di CO2, determinate da un equilibrio fra fotosintesi e respirazione della biosfera e scambi fra atmosfera e oceano, erano molto stabili, attorno alle 270-280 ppm (parti per milione). Da allora a causa di un uso sempre crescente di combustibili fossili (petrolio, carbone e gas naturale) sono aumentate a valori di circa $410 \mathrm{ppm}$, un aumento di circa $45 \%$ in 150 anni cioè di una velocità tale che il pianeta non ha mai visto nel corso dell'ultimo milione di anni [2]. Anche le concentrazioni di $\mathrm{CH} 4$ sono state relativamente costanti nel corso degli ultimi 10,000 anni, circa 750 ppb (parti per miliardo), ma sono cresciute velocemente soprattutto dalla metà del ventesimo secolo per lo più a causa dello svilupparsi di allevamenti intensivi, fino a raggiungere concentrazioni di $1700-1800$ ppb, quindi più che raddoppiate in cento anni, anche questo un andamento di velocità inedita nell'ultimo milione di anni. L'aumento rapido delle concentrazioni di que- 
sti gas serra dovrebbe quindi portare ad un aumento dell'effetto serra descritto sopra, e quindi ad un riscaldamento del clima.

Il terzo fattore è quello della variabilità naturale del sistema climatico, che è un sistema complesso con carattere caotico e quindi caratterizzato da una sua variabilità interna. Quindi, in linea di principio, il riscaldamento osservato potrebbe essere il semplice risultato di una variazione interna del sistema climatico.

Come si fa a determinare quale di questi tre fattori sta causando il riscaldamento globale? L'effetto dei forzanti naturali ed antropici può essere valutato inserendo la variazione di questi forzanti nei modelli climatici ed andando a studiare quale di questi forzanti consente ai modelli di riprodurre il riscaldamento in atto. Da specificare che i modelli climatici sono essenzialmente delle descrizioni matematiche dei processi che determinano l'evoluzione del clima, e informazioni di base sui questi modelli possono essere trovate, per esempio, in [10],[11]. Questo esercizio modellistico è stato fatto da decine di laboratori nel mondo ed ha dimostrato in maniera chiara che l'unico modo di spiegare il riscaldamento osservato dalla metà del 20 mo secolo è di introdurre nei modelli l'aumento dei gas serra di origine antropica [12]. Questo perchè, essenzialmente, negli ultimi 50 anni l'intensità della radiazione solare è stata abbastanza costante e quindi non può spiegare il riscaldamento. In più l'effetto delle grandi eruzioni vulcaniche è di raffreddare il clima più che riscaldarlo, a causa della riflessione della luce solare da parte del particolato atmosferico. Questi esperimenti hanno quindi eliminato uno dei possibili fattori causa del riscaldamento, e cioè quello dei forzanti esterni naturali.

Benchè l'aumento delle concentrazioni di gas serra di origine antropica può spiegare il riscaldamento osservato, rimane sempre in piedi l'ipotesi di una variazione naturale del clima. Per valutare questa ipotesi si può confrontare l'andamento delle temperature attuali con quelle dell'ultimo periodo caldo interglaciale (ultimi 11,300 ani), che chiamiamo Olocene. Questo confronto è riportato in Fig. 2, che presenta la ricostruzione più accurata diponibile delle temperature globali durante l'Olocene, ricostruzione che viene fatta sulla base di diversi indicatori, come le analisi di carotaggi di ghiaccio in varie zone del globo, sedimenti marini e lacustri, etc. [3]. La Fig. 2 ci mostra chiaramente come l'aumento della temperatura globale nell'ultimo secolo sia estremamente anomalo (e quindi improbabile) rispetto a quanto accaduto negli ultimi 11,300 anni e che quindi questo aumento sia difficilmente spiegabile come una fluttuazione naturale del clima. 


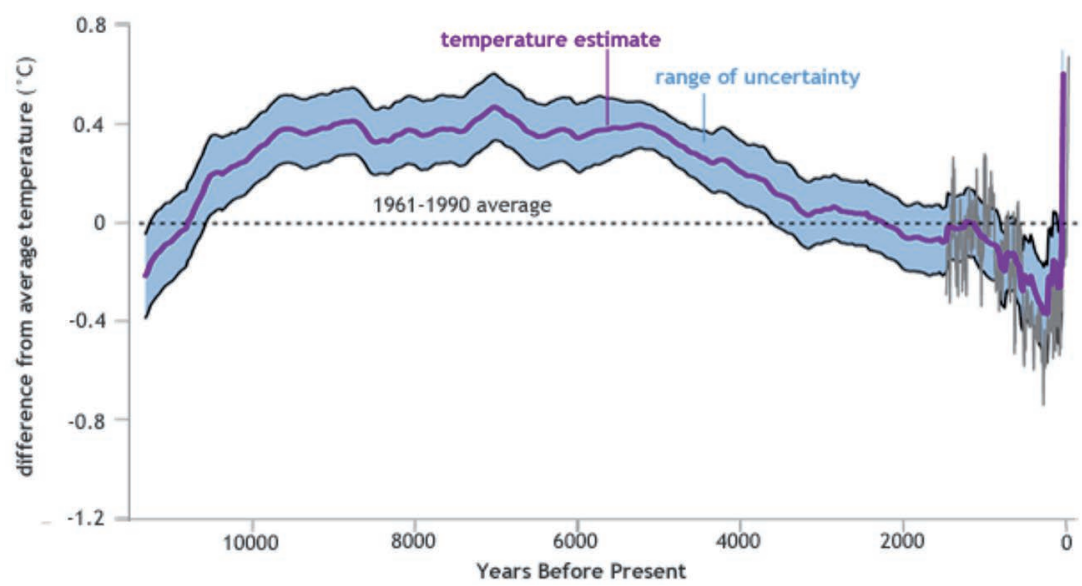

Fig. 2. Stime delle anomalie di temperatura globale superficiale (rispetto alla media di riferimento 1961-1990) nell'Olocene (ultimi 11300 anni). La curva centrale indica il valore medio della stima e le curve che delimitano la zona grigia l'intervallo di incertezza su queste stime (Cortesia della NOAA, dati da [3])

Tutte queste considerazioni hanno portato la comunità scientifica a concludere con un alto grado di certezza (95\%) che la maggior aprte del riscaldamento osservato soprattutto dalla seconda metà del $20 \mathrm{mo}$ secolo è dovuto all'aumento delle concentrazioni di gas serra di origine antropica [2].

\section{COSA POSSIAMO ASPETTARCI PER IL FUTURO ?}

Se i gas serra di origine antropica hanno un effetto così importante sul clima globale, cosa possiamo aspettarci per il futuro? Previsioni esatte su come possa cambiare il clima nel $21 \mathrm{mo}$ secolo non sono possibili, sia per la natura caotica del sistema climatico che per la impredicibilità delle emissioni future di gas serra, che dipendono dallo sviluppo socioeconomico della società. Quello che si può fare però, è di costruire delle ipotesi, o scenari, plausibili di sviluppo socioeconomico, e quindi di emissioni e concentrazioni di gas serra, e di immettere questi scenari nei modelli climatici. I modelli possono quindi essere usati per simulare la risposta del sistema climatico nel tempo a questi scenari di concentrazioni di gas serra, producendo quindi scenari di cambiamento climatico futuro. 
La comunità scientifica ha elaborato una serie di scenari di concentrazioni di gas serra per il $21 \mathrm{mo}$ secolo e di relativa risposta del sistema climatico. La Fig. 3 mostra l'aumento di temperatura globale fino al 2100 associato a due scenari particolari, RCP2.6 and RCP8.5 [2]. Nel RCP2.6, le temperature globali si stabilizzano attorno a $1^{\circ} \mathrm{C}$ in più delle temperature attuali, e quindi circa $2^{\circ} \mathrm{C}$ maggiori di quelle pre-industriali. Questo scenario quindi corrisponde approssimativamente all'obiettivo identificato nel'accordo di Parigi del 2015. Lo scenario RCP8.5, spesso chiamato il "business as usual", è il più estremo, e corrisponde ad uno scenario in cui l'uso di combustibili fossili continui ad aumentare al ritmo attuale. In questo caso i modelli climatici prevedono un riscaldamento globale fino a $4-5^{\circ} \mathrm{C}$ rispetto alle temperature attuali. Se poniamo questo valore nel contesto dell'andamento delle temperature mostrato in Fig. 2 ci possiamo rendere conto che un riscaldamento di $4-5$ gradi in cento anni è un valore assolutamente enorme, di entità mai vista nella storia recente del pianeta, e le cui conseguenze, per un sistema complesso come quello del pianeta terra, sono molto difficili da valutare, ma porterebbero sicuramente ad uno sconvolgimento del sistema climatico presente.

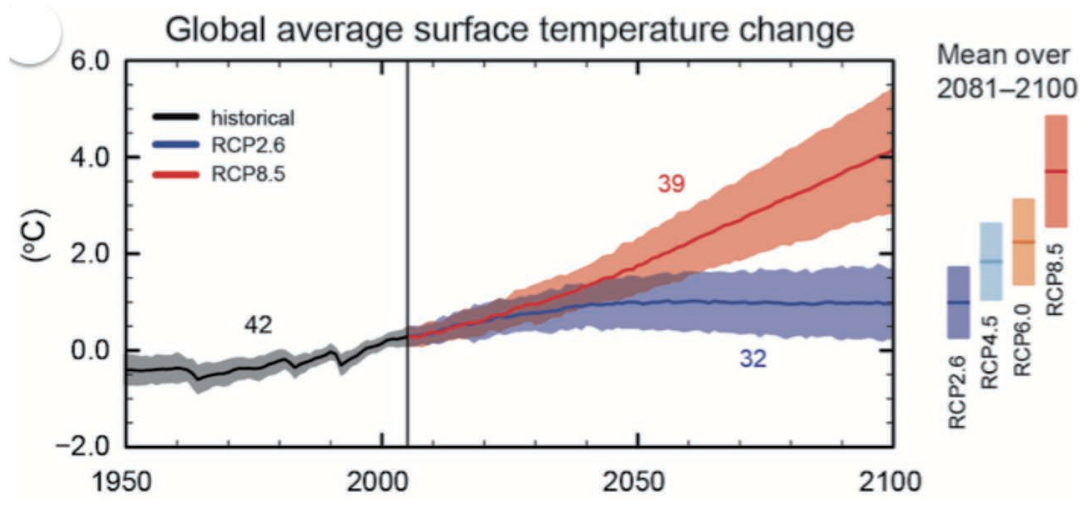

Fig. 3. Cambiamento di temperatura superficiale globale rispetto alla media per il 19862005 come simulato da proiezioni con modelli climatici globali per gli scenari RCP8.5 ("Business as usual") e RCP2.6 ("Accordo di Parigi") . Il numero per ogni curva indica il numero di modelli climatici utilizzati, la zona ombreggiata indica lo spread dei risultati dei modelli e la curva al centro di questa zona indica il valore medio. A lato sono anche riportati i valori di riscaldamento globale per il periodo 2081-2100 (media e spread dei modelli), includendo anche gli scenari intermedi RCP4.5 ed RCP6.0 (Cortesia dell'IPCC; Figura SPM.7, pannello (a, referenza [2])

Con questa premessa, è però utile identificare alcuni dei cambiamenti climatici associati al riscaldamento globale mostrato dalle Figg. 1- 
3. Il primo è il fatto che la risposta al riscaldamento globale non è uniforme attraverso il globo, ma alcune zone sono molto più sensibili di altre, $\mathrm{e}$ per questo vengono spesso chiamate "Hot-spots" (o zone calde [13]). La zona calda per eccellenza è l'Artico, dove le temperature stanno crescendo ad una velocità più che doppia di quella della media globale [7]. Questo è dovuto soprattutto allo scioglimento dei ghiacci, che diminuisce la riflettività della superficie, aumenta l'assorbimento della radiazione solare e quindi amplifica il riscaldamento. Altre zone calde sono legate alle modifiche della circolazione generale amosferica innescate dal riscaldamento globale. Una di queste consiste nello spostamento verso i poli delle traiettorie principali delle perturbazioni, cosa che produce un aumento di precipitazioni a medie e alte latitudini, dove già adesso le precipitazioni sono abbondanti, ed una diminuzione nelle zone sub-tropicali oggi già più secche. In particolare, a causa di questo fenomeno, che è peraltro già in atto, nell'area del Mediterraneo si prevede un clima molto più secco e caldo di quello odierno, il che fa del Mediterraneo un'altra delle principali zone calde del pianeta [14]. L'importanza della presenza di Hot-spots climatiche deriva dal fatto che se queste si trovano in aree con paesi particolarmente poveri, e quindi vulnerabili, possono potenzialmente creare un problema di migrazioni di massa dei cosidetti "migranti climatici" in cerca di nicchie ambientali migliori in cui vivere.

Una seconda importante risposta del clima al riscaldamento globale è la cosidetta "intensificazione del ciclo idrologico" [15]. Una atmosfera più calda è anche più energetica, e per la legge di ClausiusClapeyron, può contenere più vapor d'acqua. In queste condizioni, quando si innesca un fenomeno piovoso tenderà a piovere più intensamente. Al tempo stesso i modelli ci dicono che occorre un tempo maggiore per raggiungere le condizioni di pioggia, cioè che si allungano $i$ periodi secchi fra un evento piovoso e l'altro. In altre parole, in un atmosfera più calda piove meno frequentemente ma più intensamente. Questo fenomeno quindi risulta in un maggiore rischio che abbiano luogo eventi estremi sia di carattere alluvionale che siccitoso. In aggiunta, il riscaldamento globale aumenta anche la frequenza ed intensità di ondate di calore. L'aumento di eventi meteoclimatici estremi è in corso in molte aree del globo, e per esempio dati delle compagnie di assicurazione mostrano come il numero di eventi meteoclimatici definiti come catastrofali sia aumentato da circa 200 all'anno negli anni ottanta a circa 800 all'anno negli ultimi anni, cioè un aumento di un fattore 4 in circa 40 anni. 
La fusione dei ghiacci continentali e l'innalzamento del livello marino che ne risulta sono fra i temi più importanti nell'ambito delle problematiche legate ai cambiamenti climatici. Per lo scenario business as usual si prevede che molti ghiacciai possano diminuire notevolmente di massa o addirittura scomparire, come nel caso dei ghiacciai Alpini [8]. Se da un lato questo avrebbe impatti molto deleteri sugli ecosistemi montani, dall'altro costituirebbe anche una forte riduzione della disponibilità di acqua dolce per usi agricoli, industriali e domestici, dato che i ghiacciai oggi contengono circa il $65 \%$ dell'acqua dolce disponibile sul pianeta. Basti pensare all'importanza dei ghiacci dell'Imalaya, le cosidette Torri D'acqua Asiatiche, che sono vitali per miliardi di persone in Asia meridionale e orientale.

Riguardo l'innalzamento del livello del mare, i modelli finora prevedono fino ad un ulteriore metro per gli scenari più estremi [2]. Questa stima però è destinata a salire soprattutto perchè i ghiacci della Groenlandia stanno fondendo ad una velocità molto maggiore di quella prevista fino ad ora, probabilmente a causa dello scurimento dei ghiacci (e quindi aumento dell'assorbimento di luce solare) causato dall'inquinamento prodotto in Europa, Nord America ed Asia [6]. L'innalzamento del livello del mare ha molte conseguenze sugli ambienti naturali ed urbani costieri, come erosione delle coste, maggior frequenza ad intensità di alluvioni costieri e mareggiate, intrusione di acqua salina nel suolo. Se consideriamo che una grande porzione della popolazione mondiale vive in ambienti costieri, soprattutto in grandi megalopoli di paesi relativamente poveri, e quindi in condizioni spesso degradate, ci rendiamo conto di come questo problema sia uno dei più importanti in termini di impatti socioeconomici.

Chiaramente, tutti gli effetti climatici del riscaldamento globale menzionati fino ad ora, insieme ad altri come l'aumento della variabilità interannuale, hanno impatti su molteplici settori di importanza per la società, per esempio salute, trasporti, turismo, agricoltura, gestione delle risorse idriche, biodiversità, inquinamento di aria e acqua, e così via. Ognuno di questi richiederebbe un trattamento separato ben al di là degli scopi di questo contributo. È importante però menzionare il problema dei cosidetti "punti di non ritorno", o "tipping points" [16] Questi sono processi i quali, una volta innescati e superata una certa soglia, avrebbero un carattere sostanzialmente semi-irreversibile, almeno su scale temporali di interesse alla società umana, e porterebbero a mutamenti molto profondi del sistema climatico. 
La comunità scientifica si è soffermata soprattutto su tre di questi "tipping points" [2]. Il primo è lo scioglimento dei ghiacci della Groenlandia, di cui abbiamo già parlato. È stato valutato che se l'intera copertura dei ghiacci della Groenlandia fondesse, questo porterebbe ad un aumento del livello del mare globale di superiore ai 7 metri, con conseguenze la cui gravità è facilmente immaginabile. Il secondo tipping point è il cosidetto collasso della circolazione globale oceanica termoclina. Gli oceani del globo sono collegati fra loro da una circolazione profonda che viene sostanzialmente sostenuta dalla formazione di acque molto dense e fredde nel nord Atlantico, come risultato della formazione dei ghiacci artici. Questa circolazione per esempio porta alla formazione della Corrente del Golfo, che mantiene il clima dell'Europa relativamente mite. Un riscaldamento globale che riducesse o addirittura eliminasse la formazione dei ghiacci Artici potrebbe indebolire notevolemnte questa circolazione fino al punto da farla interamente collassare, e questo porterebbe ad uno sconvolgimento generale della circolazione oceanica ed atmosferica globale. Il terzo tipping point è la scomparsa della foresta Amazzonica, legata al fatto che i modelli predicono una forte riduzione delle precipitazione nel bacino dell'Amazzonia. Questo ovviamente porterebbe anche ad enormi modifiche del sistema climatico. Fortunatamente nessun modello predice che questi tipping points siano stati già raggiunti, o vengano raggiunti nel corso del $21 \mathrm{mo}$ secolo, anche negli scenari più estremi. Questo però potrebbe accadere nel corso di qualche centinaio di anni nel caso di un riscaldamento sostenuto di diversi gradi. A quel punto, il clima del pianeta, e quindi il pianeta stesso, sarebbe molto diverso da come è ora.

È chiaro dagli esempi riportati in questa sezione che il riscaldamento globale potrebbe potenzialmente portare a sconvolgimenti tali del sistema climatico da mettere a repentaglio lo sviluppo sistenibile della società umana, almeno come adesso la conosciamo. Questo però può essere evitato con delle politiche efficaci di cosidetta mitigazione e adattamento, che sono l'oggetto della prossima sezione di questo contributo.

\section{COSA SI PUÒ FARE PER AFFRONTARE IL PROBLEMA DEI CAMBIAMENTI CLIMATICI?}

Nell'accordo di Parigi del 2015 si è stipulato che politiche debbano essere intraprese dai governi del mondo per limitare il riscaldamento 
globale al di sotto della soglia di $2{ }^{\circ} \mathrm{C}$ rispetto ai valori pre-industriali, e quindi poco meno di $1^{\circ} \mathrm{C}$ rispetto ai valori attuali. Questa soglia dei 2 "C è stata individuata come cosidetta "soglia di pericolo", al di là della quale i danni provocati dai cambiamenti climatici sarebbero difficilmente gestibili anche con delle efficaci politiche di adattamento, e quindi sarebbero inaccettabii per la società. Per stabilizzare le temperature globali al di sotto di questa soglia di pericolo occorre stabilizzare le concentrazioni di gas serra a valori di equivalente CO2 attorno alle 450 ppm (quindi non lontani da quelli attuali), e questo richiede una diminuzione delle emissioni, perchè i processi di rimozione dei gas serra non sono sufficienti oggi a controbilanciare le emissioni correnti. A queso processo di riduzione delle emissioni si dà il nome di "mitigazione". Nello specifico, per ottenere l'obiettivo dell'accordo di Parigi si dovrebbero ridurre le emissioni di circa $75-80 \%$ rispetto ai valori attuali entro il 2050 e raggiungere la neutralità di flussi di carbonio in atmosfera entro il 2100.

Dunque, anche nel caso si ottenesse l'obiettivo dell'accordo di Parigi, dovremmo aspettarci un ulteriore livello di riscaldamento globale ed associati cambiamenti climatici, i cui impatti devono essere gestiti attraverso delle politiche di cosidetto "adattamento". Un esempio tipico di adattamento è la costruzione di barriere costiere più alte in risposta all'aumento del livello del mare. Un altro è l'implementazione di modifiche nella gestione delle risorse idriche o nella pianificazione territoriale in relazione a modifiche del ciclo idrologico terrestre. L'adattamento è una forma di risposta al riscaldamento globale di carattere locale, perchè dipende dagli impatti che i cambiamenti climatici hanno sulle diverse realtà territoriali.

La mitigazione invece ha un carattere globale. $\mathrm{CO} 2$ e $\mathrm{CH} 4$ hanno un tempo di vita molto lungo in atmosfera, dai 50 ai 100 anni, e quindi sono ben mescolati nel globo. Questo vuol dire che non basta che sia un paese ad implementare politiche efficaci di mitigazione, ma devono contribuire tutti i paesi del mondo. Come abbiamo visto, la sorgente antropica principale di CO2 è l'uso di combustibili fossili, che oggi copre circa 1' $80 \%$ del fabbisogno energetico globale. Quindi, essenzialmente, il problema della mitigazione per il $\mathrm{CO} 2$ è un problema di decarbonizzazione del sistema energetico. Un primo fondamentale passo in questo contesto è quello di un aumento dell'efficienza energetica. Oggi globalmente si spreca circa il 60-65\% dell'energia prodotta, al momento della produzione, trasporto e uso di questa energia. Quindi 
una maggiore efficienza energetica darebbe già un contributo fondamentale alla riduzione delle emissioni. Un modo per aumentare questa efficienza è quello di riconvertire sistemi oggi basati per lo più su motori termici (trasporto, riscaldamento, industria) in sistemi elettrici, perchè questi ultimi hanno un rendimento molto maggiore.

Questa elettrificazione però deve essere supportata da una produzione di energia elettrica basata su fonti alternative. A questo riguardo, fonti rinnovabili, come idroelettrico, geotermico, eolico, solare, biomasse e, di recente sviluppo, onde marine, offrono una alternativa ai combustibili fossili sempre più conveniente, sia dal punto di vista economico che ambientale. Per esempio, un indicatore tipico usato per misurare la convenienza di una forma di energia è l'EROEI (Energy return ver Energy Invested), che misura il rapporto fra energia prodotta da una certa sorgente e quella spesa per produrla. Quindi maggiore è l'EROEI più conveniente è una forma di energia. L'EROEI oggi è dello stesso ordine, se non maggiore, per molte forme di energia rinnovabile che per le fonti fossili [17], e mentre l'EROEI per le rinnovabili cresce con il migliorare delle tecnologie, quello delle fonti fossili diminuisce per le maggiori difficoltà di estrarre e raffinare i combustibili. Altri meccanismi di mitigazione includono la riduzione di allevamenti intensivi, che sono la maggiore fonte di $\mathrm{CH} 4$ di origine antropica, e la riduzione della deforestazione, che oggi contribuisce per circa 10\% di emissoni equivalenti di $\mathrm{CO} 2$, con eventuale inizio di riforestazione a livello globale.

\section{CONSIDERAZIONI CONCLUSIVE}

In questo contributo abbiamo esaminato i punti principali riguardanti il riscaldamento globale. Abbiamo visto come l'evidenza è ormai conclusiva che siamo in un trend di riscaldamento globale (poco più di $1{ }^{\circ} \mathrm{C}$ dall'inizio del $20 \mathrm{mo}$ secolo) e che questo è dovuto con un alto livello di certezza $(>95 \%)$ alle emissioni di gas serra di origine antropica, in particolare l'uso di combustibili fossili per la $\mathrm{CO} 2$ ed alcune pratiche agricole per il $\mathrm{CH} 4$. Abbiamo altresì visto che un aumento di temperatura globale di $1^{\circ} \mathrm{C}$ in 120 anni è di una rapidità tale che non è mai occorsa almeno negli ultimi 11,300 anni, ed è quindi un evento molto anomalo per il pianeta. Proiezioni per il $21 \mathrm{mo}$ secolo producono una 
forchetta che va da un ulteriore grado di riscaldamento entro il 2100 nello scenario meno impattante a $4-5^{\circ} \mathrm{C}$ per il più estremo, il che produrrebbe un vero sconvolgimento nel sistema climatico del pianeta.

Fra i cambiamenti climatici legati al riscaldamento globale abbiamo messo in evidenza la presenza di zone climatiche particolarmente sensibili (hotspots), come l'Artico, il Mediterraneo ed il bacino dell'Amazzonia; l'intensificazione del ciclo idrologico con conseguente aumento di frequenza ed intensità di eventi meteoclimatici estremi; la riduzione dei ghiacciai, con conseguente perdità di risorse idriche usufruibili; l'aumento del livello del mare, con conseguente danneggiamento, o addirittura scomparsa, di aree costiere. Ovviamente però molti altri elementi del sistema climatico ed importanti settori socioeconomici sarebbero influenzati, come la biodiversità, l'agricoltura, l'inquinamento, il turismo e così via.

Infine abbiamo dato alcuni esempi di politiche di adattamento e di strategie di mitigazione necessari a contrastare il riscaldamento globale. Fra queste ultime, in particolare, la conversione da un sistema energetico prevalentemente basato sull'uso di combustili fossili ad uno in cui l'efficienza energetica sia maggiormente curata, per esempio tramite l'elettrificazione di settori come i trasporti, il riscaldamento e l'industria, e l'energia elettrica sia prodotta attraverso fonti rinnovabili che stanno diventando sempre più competitive.

Chiaramente esistono ancora molte aree che necessitano di maggiore ricerca. Nell'ambito degli aspetti fisici del problema, I modelli climatici devono essere migliorati, soprattutto nella loro rappresentazione di processi legati a nubi e precipitazione, per aumentare l'affidabilità e diminuire le incertezze delle simulazioni di clima futuro. Questo necessita anche di migliori osservazioni per validare i modelli. Le simulazioni di cambiamento climatico a livello locale e regionale sono ancora caratterizzate da un alto grado di incertezza, anche per la bassa risoluzione dei modelli climatici globali che non consente di rappresentare adeguatamente processi a scale regionali e locali. Diverse tecniche sono state sviluppate per migliorare questo aspetto della modellistica, come l'uso di modelli regionali ad alta risoluzione [18] o di tecniche di downscaling statistico [19], ma sicuramente occorre molta ricerca in questo campo. La rappresentazione delle interazioni fra biosfera, sia continentale che marina, e le altre componenti del sistema climatico deve anche essere migliorata, data la sua importanza nel determinare una serie di feedbacks che possono marcatamente amplificare il riscaldamento glo- 
bale. Ed infine, menzionerei la criosfera, la cui risposta al riscaldamento globale è fondamentale nel determinare l'entità e gli effetti dei cambiamenti climatici e per cui alcuni processi sono ancora non chiari (come lo scioglimento veloce dei ghiacci della Groenlandia).

A dispetto di queste incertezze, è chiaro sulla base della ricerca scientifica condotta nelle ultime 3 decadi, che quello del riscaldamento globale è un problema fondamentale per lo sviluppo sostenibile della società umana come oggi la conosciamo. È un problema però che può essere affrontato e tenuto sotto controllo con delle appropriate strategie di mitigazione. Purtroppo ad oggi le negoziazioni internazionali su queste strategie hanno fallito nel produrre accordi vincolanti, dato che l'accordo di Parigi, per quanto importante nello stabilire un obiettivo preciso, non è appunto legalmente vincolante. Esiste un forte elemento di urgenza nell'implementazione di strategie di mitigazione, perchè le azioni intraprese ora avranno effetti sul pianeta per decadi a venire dato il lungo tempo di permanenza dei gas serra in atmosfera e data l'inerzia di alcune componenti del sistema climatico (e.g. gli oceani). È quindi necessario che una forte spinta dal basso, dalla società civile, riesca a dare l'impeto necessario per implementare azioni tempestive ed efficaci per contrastare il riscaldamento globale.

\section{REFERENCES}

[1] Crutzen PJ, The Anthropocene, Earth System Science in the Anthropocene, 2006: 13-18.

[2] IPCC 2013. Climate Change 2013: The Physical Science Basis. Contribution of Working Group I to the Fifth Assessment Report of the Intergovernmental Panel on Climate Change [Stocker TF, et al., Eds.], Cambridge University press, Cambridge, UK, 2013.

[3] Marcott SA, Shakun JD, Clark PU, Mix AC, A reconstruction of regional and global temperature for the past 11,300 years, Science, 339: 1198-1201.

[4] Giorgi F, L’Uomo e la Farfalla. Sei domande su cui riflettere per comprendere i cambiamenti climatici, FrancoAngeli Ed., 2018, 148pp.

[5] Vaughn DG, Comiso JC, et al. , Observation: Cryosphere, in Climate Change 2013: The Physical Science Basis. Contribution of Working Group I to the Fifth Assessment Report of the Intergovernmental Panel on Climate Change [Stocker TF, et al., Eds.], Cambridge University press, Cambridge, UK, 2013, 317-382.

[6] Chen JL, Wilson CL, Tapley BD, Satellite gravity measurements confirm accelerated melting of Greenland Ice Sheet, Science, 313: 1958-1960.

[7] Rott H, Muller F, Nagler T, Floricioiu D, The imbalance of glaciers after disin- 
tegration of larsen-B ice Shelf , Antarctic Peninsula, The Cryosphere, 5:125-134.

[8] IPCC 2019, Special report on the ocean and cryosphere in a changing climate. Intergovernmental Panel on Climate Change, Cambridge University press, Cambridge, UK, 2019.

[9] Santer BD, et al. Tropospheric warming over the past two decades, Scientific Reports, 2017, Art. No. 2336.

[10] MacGuffie K, Henderson-Sellers A, The Climate Modeling Primer, 4th Edition, John Wiley and Sons, New York, USA, 2014, 231 pp.

[11] Washington W, Parkinson CL, An Introduction to Three-Dimensional Climate Modeling, University Science Books, 2005, 354 pp.

[12] Bindoff, NL, et al. , Detection and attribution of climate change: From global to regional, Chapter 10 of Climate Change 2013: The Physical Science Basis. Contribution of Working Group I to the Fifth Assessment Report of the Intergovernmental Panel on Climate Change [Stocker TF, et al., Eds.], Cambridge University press, Cambridge, UK, 2013, 867-952.

[13] Giorgi F, Climate change hotspots, Geophysical Research Letters, 2006, 33 , doi:10.1029/2006GL025734.

[14] Giorgi F, Lionello P, Climate change projections for the Mediterranean region, Global and Planetary Change, 2008, 63, 90-104.

[15] Giorgi F, Raffaele F, Coppola E, The response of precipitation characteristics to global warming from climate projections, Earth System Dynamics, 10:73-89.

[16] Lenton TM, et al., Climate tipping points - too risky to bet against, Nature, 2019, 575: 592-596.

[17] Hall CAS, Lambert GJ, Balogh SB, EROEI of different fuels and the implications for society, Energy Policy, 64:141-152.

[18] Giorgi F, Thirty years of regional climate modeling: Where are we and where are we going?, Journal of Geophysical research - Atmospheres, 124:5696-5723.

[19] Wilby RL, et al., Statistical downscaling of general circulaiton model output: A comparison of methods, Water Resources Research, 34:2995-3008. 
